Closer liaison between dentistry and medicine is increasingly relevant.

The author presents an intriguing possibility which will strengthen that relationship.

Readers are invited to respond.

\title{
Physician and Dental Surgeon's roles in diagnosing hypertension in association with Lichen Planus and Geographic Tongue - the perspective of a Clinician
}

SADJ November 2019, Vol. 74 No. 10 p528 - p529

VK Vaishnavi Vedam', G Sivadas ${ }^{2}$

Dear Editor,

Hypertension is a serious medical condition resulting in a high degree of mortality if undiagnosed and untreated in the early stages of its progression. Although public awareness of this cardiac manifestation has increased, asymptomatic cases still go undetected and untreated, resulting in complications.

The General Physician (GP) is the first line of interaction with patients presenting varying degrees of hypertension. The Dental Surgeon (DS) encounters a wide range of patients with oral manifestations of Lichen Planus and Geographic Tongue (Benign migratory glossitis, erythema migrans).

Liaison between the GP and DS in the management of these patients is rare as they usually limit themselves to the principal problems of patients. However, current researchers suggest a possible link between the occurrence of Lichen Planus, Geographic tongue and hypertension in adults.

Cardiovascular Diseases (CVD) have over the last few decades dominated the Malaysian National Health Survey as being the leading cause of morbidity and

Author affiliation:

1. Venkata K Vaishnavi Vedam: MDS, Senior lecturer, Faculty of Dentistry, Asian Institute of Medicine, Science and Technology (AIMST) University, Malaysia.

2. Ganapathy SIvadas: MDS, Senior lecturer, Department of Pediatric Dentistry, Faculty of Dentistry, Asian Institute of Medicine, Science and Technology (AIMST) University, Malaysia.

Corresponding author: Venkata K Vaishnavi Vedam

Faculty of Dentistry Asian Institute of Medicine, Science and Technology (AIMST) University, Malaysia.

Email: vaishnavivedam@gmail.com

Author contributions:

1. Venkata K Vaishnavi Vedam: Primary author - $60 \%$

2. Ganapathy SIvadas: Secondary author $-40 \%$ mortality and that statistic is likely to continue. Of all the risk factors contributing to CVD, hypertension poses the greatest risk for all genders, based on the latest literature. ${ }^{2}$

The relationship between high blood pressure (hypertension) and the risk of cardiovascular events is continuous, consistent and independent of other risk factors and it may lead to myocardial infarction, heart failure, stroke and kidney diseases.

The latest National Health and Morbidity Survey (NHMS) for risk factors for non-communicable diseases (NCD) showed an overall prevalence of hypertension of $35.3 \%$ among adults 18 years and above. ${ }^{2}$

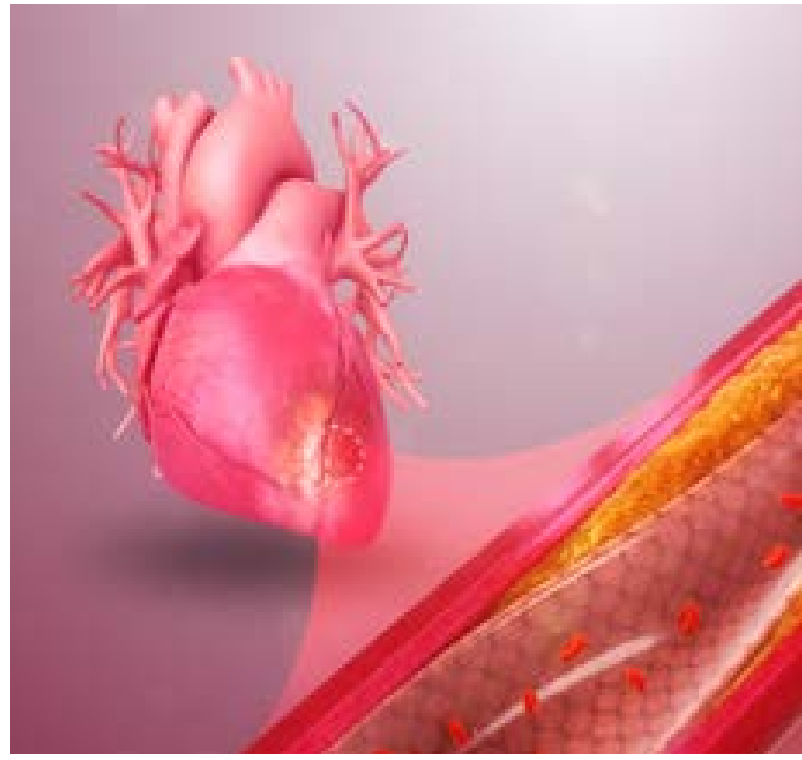

Image Source. http://www.scientificanimations.com [CC BY-SA 4.0]. (https://creativecommons.org/licenses/by-sa/4.0). 
Lichen Planus and Geographic tongue in hypertensive patients have been reported infrequently in the literature. Lichen Planus (a chronic inflammatory disorder) and hypertension may interchangeably contribute as risk factors with alterations in the underlying immune system.

Notably, Lichen Planus could have occurred as an adverse reaction to the Anti-hypertensive ACE Inhibitor drugs and Statins. ${ }^{3}$ Reports also suggest that in chronic inflammation, reactive oxygen species and cytokines (Tumour necrosis factor TNF, Interleukins IL-2, IL6) are released during keratinocyte degeneration in the pathogenesis of lichen planus. These may be implicated in the rise of dyslipidemia in hypertensive patients. ${ }^{4}$

Geographic tongue is an asymptomatic inflammatory disorder of the tongue of uncertain aetiology, but which may be associated with several factors such as vitamin deficiency, congenital anomaly, asthma, systemic diseases such as psoriasis, anaemia, gastrointestinal disturbances, candidiasis, hormonal imbalance and psychological conditions. ${ }^{5}$

Some of these, such as anaemia and vitamin deficiency, are secondary to underlying hypertension and may result in the development of Geographic tongue. Cases linking the occurrence of Lichen Planus or Geographic tongue with hypertension may have been under-reported in the Malaysian population, but that does not weaken the importance of the present discussion.

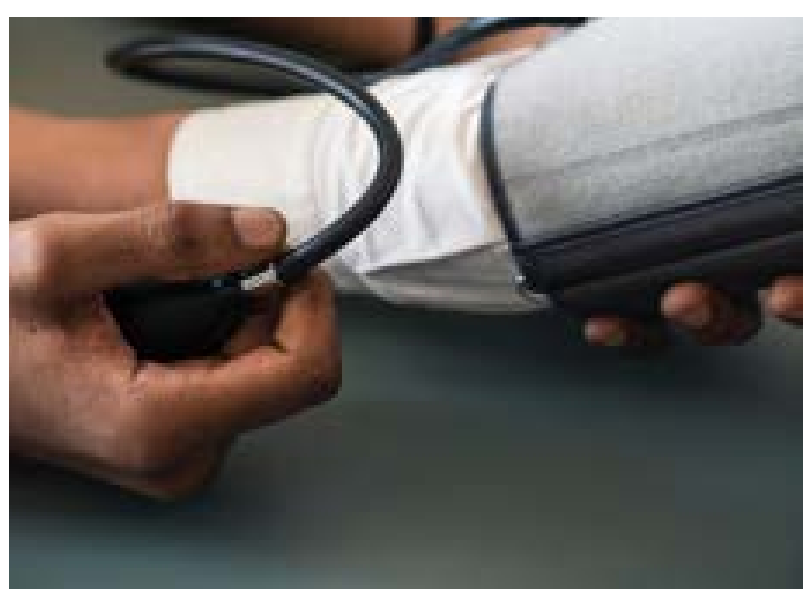

In view of the rise in the CVD mortality cases with hypertension in adults, the authors believe new associations may pave the way to a potential strategy for General Physicians and Dental Surgeons to identify hypertension at the earliest stages, decreasing the number of missed diagnoses.

An integrated approach is essential in routine clinical practice in the diagnosis, either directly or indirectly, of patients involved with hypertension and/or CVD. An awareness of the clinical and oral manifestations observed in the patient may lead to an effective and early diagnosis.

Both the General Physician (GP) and the Dental Surgeon (DS) could reduce the prevalence of the national problem by diagnosing undetected hypertensive cases and, indirectly, the CVD cases, with the help of the associated, although not common, oral manifestation of Lichen Planus or Geographic tongue.

Yours faithfully,

\section{Dr VK Vaishnavi Vedam \& Dr G Sivadas}

\section{Declaration}

The authors declare no conflict of interest.

\section{References}

1. Kumar P, Mastan KMK, Chowdhary R, Shanmugam K. Oral manifestations in hypertensive patients: A clinical study. Journal of Oral and Maxillofacial Pathology 2012; 16(2): 215-21.

2. Management of Hypertension. Clinical Practice Guidelines, Malaysian Society of Hypertension, $5^{\text {th }}$ edition; 2018: 26-27. $\mathrm{MOH} / \mathrm{P} / \mathrm{PAK}$ 391.18(GU).

3. Farzin M, Derafshi R, Ghapanchi J, Kafsh AZ Rezaiee M. Oral manifestations of hypertension and rheumatic heart disease: a cross sectional study in elderly patients. Asian Journal of Medical and Pharmaceutical Researches 2016; 6(2): 09-13.

4. Arias-Santiago S, Buendía-Eisman A, Aneiros-Fernández J, Girón-Prieto MS et al. Cardiovascular risk factors in patients with Lichen Planus The American Journal of Medicine 2011; 124, 543-8.

5. Shah N, Kariya P, Dave B, Thomas P. Geographic Tongue: A case report with review of literature. Adv Hum Biol. 2016; 6: $142-4$. 


\section{What are some of the key inputs that we as the dental profession (Dentists, Therapists, Hygienists, Technicians, Specialist and Assistants) should agree upon and aim to achieve under the $\mathrm{NHI}$ ?}

- an opinion piece submitted to the SADJ

SADJ November 2019, Vol. 74 No. 10 p530 - p531

\section{J Mthethwa}

Dear Editor,

I am aware that the NHI Bill is out there with a deadline for comments by the end of November. I have decided to pen down some of my personal views and opinions on how we as the profession should go about implementing the $\mathrm{NHI}$ in this country.

These are my personal views as informed by the many years I spent in private practice as a dentist both in this country and abroad, my years as a Senior Government Official in the Province, my interaction with colleagues formally and informally and lastly my interaction with academics and students who are concerned about the future of the profession. I therefore write here in my personal capacity having perused through the summary of the Bill.

Below are some of the key principles on working arrangements on which we, as the profession, need to agree. We need to ensure that in the $\mathrm{NHI}$ environment:

- We promote a strong multidisciplinary approach. This implies that we need dentist-led teams with dental therapist, oral hygienist, dental technician and dental assistants working side by side, each freely exercising his/her scope of practice.

- We promote group practice models and discourage solo practice models.

- There are various contracting models for primary and secondary care in general dental practice and tertiary/quaternary services through Hospital care.

\section{Author affiliation:}

Jimmy Mthethwa: BDS (Medunsa), PG Cert (UK), MPH (UK), MBA

(UK), Stomatologist, Department of Oral Hygiene, Community Den-

tistry, School of Oral Health Sciences, University of Witwatersrand Johannesburg, South Africa.

Email: jimmy.mthethwa@wits.ac.za

ORCID Number: 0000-0002-3586-1930

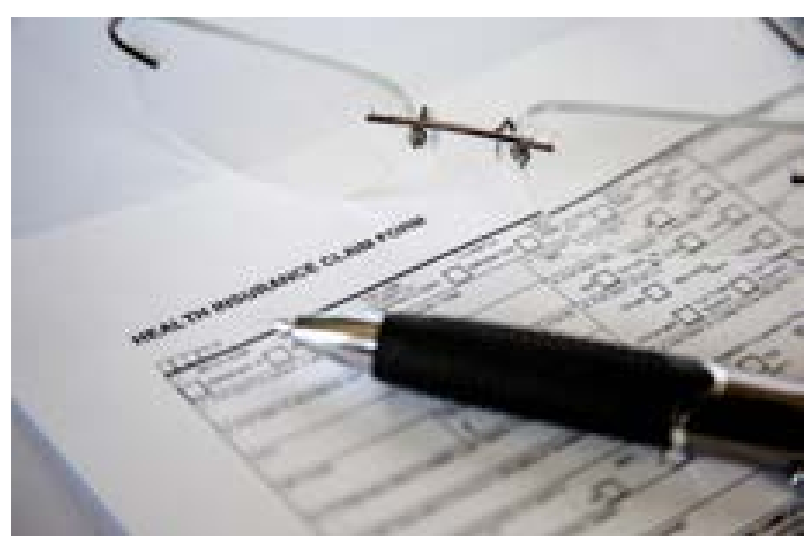

- Acceptable and cost-effective clinical protocols and guidelines to be used to guide clinicians are developed by the profession.

- There is a proper referral system to guide the profession and to be followed by patients.

- There are local contracting mechanisms of dental services within district health authority, moving away from central government.

- Dental service packages are developed for all population groups, serving children, adults, elderly, special needs patients and for prison settings.

On the key Ministerial Committees, we need to ensure that at least one or two members of the dental profession are included in each of the key committees such as:

- Benefits Advisory Committee,

- Health Benefits Pricing

- Stakeholder Advisory Committees

- Technical Committee including

- Remuneration Committees 
On the Benefits Advisory Committee, we need to ensure that:

- There is a recognition that Oral Health is not just Primary Health care alone.

- Oral Health benefits under NHI must be defined across all levels of health care from Primary to Secondary, Tertiary and to Quaternary services.

- The design of package of services will be executed by the Oral Health Profession.

- There are norms, standards and prescribed minimum benefits for Oral Health.

- Clinical protocols and guidelines for Oral Health are developed in this committee

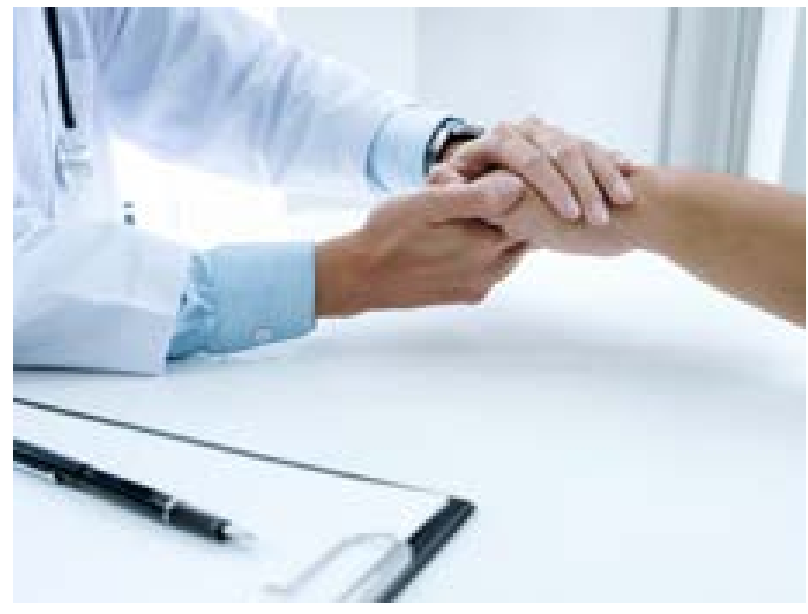

On dental Information systems, we need to ensure that:

- A universal standardized electronic clinical record system for Oral Health should be developed.

- Such a system can interface with other data bases such as the medical fraternity, Home Affairs, etc.

- Minimum data indicators are developed for Oral Health at various levels of care.

- The system should include radiology, pharmacology, laboratory and clinical record modules.

- The system must be able to interface at all levels of care, primary through to quaternary care.

- The system must be compatible with other systems and be upgradable to ensure future sustainability.

On the role of central hospitals and academic Oral Health Centres, we need to consider that:

- There are four Academic Oral Health centres in the country.

- These are classified as central/quaternary services.

- These must be under the National Department of Health and be centrally managed as semi-autonomous centres focusing on teaching and research, with service delivery on tertiary and quaternary levels only. Each centre should be allowed to raise and retain its additional revenue.

- These four centres must support the nine provinces in terms of training, research etc.

- The country needs to invest in the KZN/UKZN Dental Therapy and Oral Hygiene School, developing it into a full Dental School offering all other disciplines and training.
There is a need to consider the possibility of establishing an additional Dental School elsewhere in the country as driven by need and evidence.

These training platforms must focus on primary prevention and develop middle level professional development over and above the tertiary and quaternary focus.

Finally, there are some grey areas that I think need more attention and consensus from amongst the professions:

- The Bill needs to be clear on the eligibility of refugees and foreign nationals for Oral Health services as the current model may be unsustainable.

- The Bill needs to define Oral Health services which may be excluded from the $\mathrm{NHI}$ and may then be channeled to private medical insurance.

- The Office of Health Standards and Compliance needs to strengthen the Inspectorate for Oral Health in the inspection of dental practices and dental facilities in the hospitals to ensure appropriate equipment and settings are available.

- The Capitation vs. the Fee for Service models of reimbursement need to be balanced in a hybrid system to counter the effects of both under- and over-serving of patients.

\section{In conclusion:}

Clearly there is a lot that the profession needs to work on and develop rapidly in the coming weeks, months and years. The profession needs to put differences aside, for it can ill afford fragmentation and fighting over small pieces of the pie. We must work together as one united Oral Health Profession voice.

The NHI Bill provides the profession with an opportunity to start afresh, to put its house in order and to fix Oral Health not just for now but for the benefit of future generations to come. If all these objectives are achieved it would be easier to ensure that Government listens to one voice of Oral Health and to ensure that Oral Health is an integral part of the General Health service.

Yours faithfully,

\section{Dr Jimmy Mthethwa}

